\title{
53. The Dimorphism and the Crystal Habits of Copper-Oxinate Precipitates
}

\author{
By Eiji Suito and Eiichi Sekido \\ Institute for Chemical Research, Kyoto University \\ (Comm. by S. Horiba, M.J.A., April 12, 1957)
}

8-Hydroxyquinoline has been used in the quantitative analysis of copper. It was found ${ }^{11}$ that copper-oxinate precipitates as greenish yellow needle form at the outset and then they take a green plate form. In this study, the conditions forming these two kinds of copperoxinates and the transformation from needle to plate form were investigated from the viewpoint of chelate chemistry as well as crystallography.

The morphology by electron microscopy. It was recognized by observing electron micrographs that copper-oxinate precipitates had two types, namely the needle-like or fibrous form, which the authors named $\alpha$-form as shown in Fig. 1(a) and the plate form such as tetragonal, hexagonal, or rhombohedral form, named $\beta$-form, as shown in Fig. 1(b), and that unstable $\alpha$-form transformed into stable $\beta$-form.

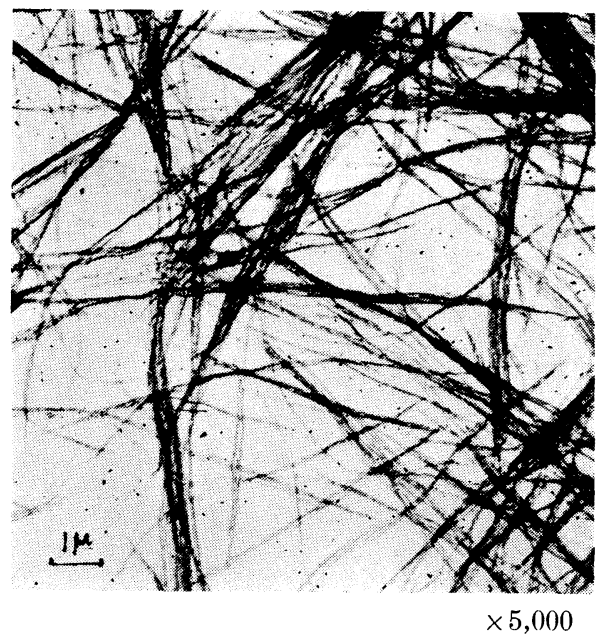

(a) $\alpha$-Form

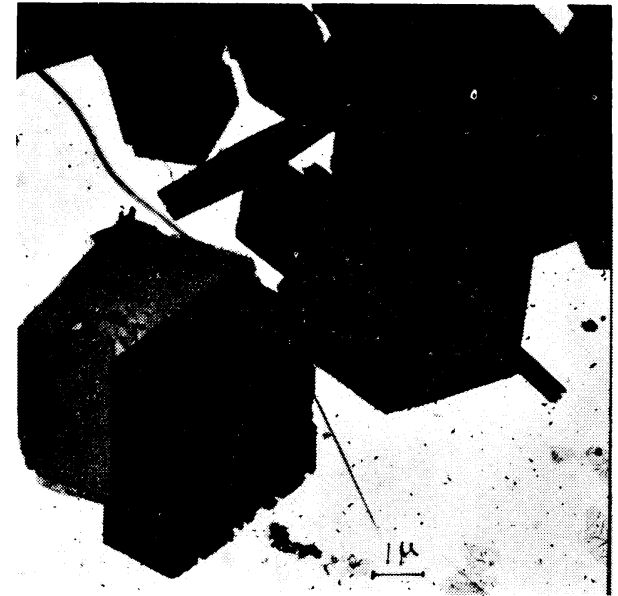

(b) $\beta$-Form

Fig. 1. Electron micrographs of two types of copper-oxinate precipitates

The relations between this dimorphism and the conditions of the precipitation are shown briefly in Table I from the results of many electron micrographs.

The influence of the solvent of oxine, namely ethyl alcohol, acetone, or acetic acid, and the influence of $\mathrm{pH}$ at the precipitation were not 
Table I. Relation between the dimorphism and the condition of the precipitation

\begin{tabular}{|c|c|c|c|}
\hline \multirow{2}{*}{$\begin{array}{c}\text { Temperature } \\
\left({ }^{\circ} \mathrm{C}\right)\end{array}$} & \multirow{2}{*}{$\begin{array}{l}\text { Conc. of } \mathrm{CuSO}_{4} \\
(\mathrm{Mole} / 1)\end{array}$} & \multicolumn{2}{|c|}{ Shape of precipitate } \\
\hline & & at the outset & after standing \\
\hline below $40^{\circ}$ & $10^{-3} \sim 10^{-8}$ & needle & plate (tetra.) \\
\hline , & above $10^{-1}$ & plate (tetra.) & no change \\
\hline above $40^{\circ}$ & $10^{-3} \sim 10^{-2}$ & $\begin{array}{l}\text { needle and } \\
\text { plate (hex. rhom.) }\end{array}$ & $\begin{array}{c}\text { slight change } \\
\text { to plate (hex. rhom.) }\end{array}$ \\
\hline , & above $10^{-1}$ & $\begin{array}{l}\text { needle and } \\
\text { plate (hex. rhom.) }\end{array}$ & plate (hex. rhom.) \\
\hline
\end{tabular}

found on the dimorphism of copper-oxinate precipitates.

Composition of the two types of copper-oxinate precipitates.

(a) Ratio of copper and oxine. Using the limited weight of copper sulphate pure $\alpha$ - and $\beta$-form copper-oxinates were prepared respectively. In the case of $\alpha$-form preparation, excess hydrogen peroxide was added to copper sulphate solution in order to prevent the transformation to $\beta$-form. The oxine in the precipitates was dissolved $1 \mathrm{~N}$ hydrochloric acid and then was determined colorimetrically by using spectrophotometer. The limited weight of $\alpha$ - and $\beta$-form copperoxinates dried in air was extracted with chloroform and then copper was determined colorimetrically. ${ }^{2)}$

It was found from the result that both $\alpha$ - and $\beta$-form copperoxinate precipitates have the same composition, that is, copper:oxine $=1: 2$.

(b) Crystal water. The crystal water of $\beta$-form copper-oxinate precipitate, sufficiently dried in air, was calculated from the weight loss with a thermobalance. The dehydration begins at about $48^{\circ} \mathrm{C}$ and the weight becomes constant above $90^{\circ} \mathrm{C}$. This weight loss corresponds to 2 moles of water per 1 mole of copper-oxinate. The same weight loss was also observed with $\alpha$-form copper-oxinate.

Crystal structure analysis by $X$-ray diffractometer. It was found that $\alpha$ - and $\beta$-form copper-oxinate precipitates had the same composition, that is, same coordination number of copper. Therefore the remaining question is a difference in crystal structure.

X-ray diffraction patterns of the precipitates, dried in the air and finely ground with mortar, were recorded on a diffractometer, using Ni filtered copper radiation $(\lambda=1.54 \AA)$. The correction of angle was performed by $\alpha$-quartz or aluminum plate. The X-ray diffraction patterns obtained with $\alpha$ - and $\beta$-form copper-oxinate 2 hydrate are shown in Fig. 2 and it is recognized that the crystal structures differ from each other. 

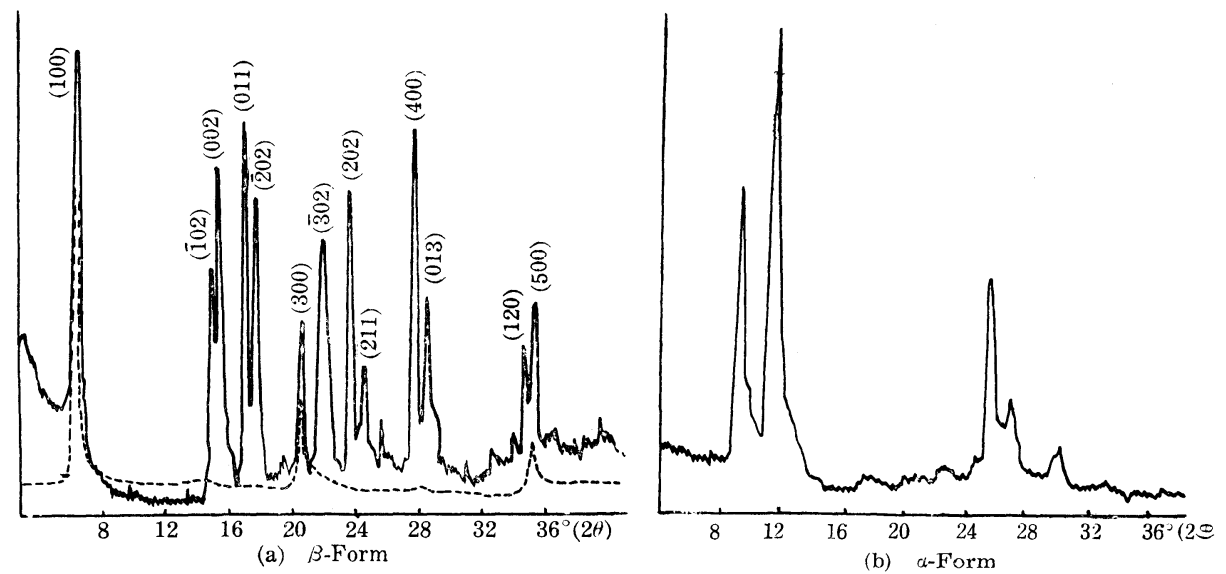

Fig. 2. The X-ray diffraction patterns of $\alpha$ - and $\beta$-form copper-oxinate precipitates

(a) $\beta$-Form copper-oxinate 2 hydrate. The crystal structure of zinc-oxinate 2 hydrate has been determined by Beritt, Cady, and Mundy. ${ }^{3)}$ Using the fact that copper-oxinate 2 hydrate is isomorphous with the corresponding zinc salt, Kruh and Dwiggins ${ }^{4}$ have reported that the lattice constants were $a=13.01, b=5.56, c=11.38 \mathrm{~A}, \beta=106.1^{\circ}$, and $Z=2$ and the space group was probably $P 2_{1} / c$. Interplanar spacings calculated on these constants had good accordance with the observed values of $\beta$-form copper-oxinate 2 hydrate in an accuracy better than $1 \%$ as shown in Table II.

Table II. Interplanar spacings of $\alpha$ - and $\beta$-modifications

\begin{tabular}{|c|c|c|c|c|c|c|c|}
\hline \multicolumn{4}{|c|}{$\beta$-Modification } & \multicolumn{4}{|c|}{$\alpha$-Modification } \\
\hline$h k l$ & $d$ (Calc.) & $d$ (Obs.) & $I$ & $h k l$ & $d$ (Calc.) & $d(\mathrm{Obs})$. & $I$ \\
\hline 100 & 12.50 & 12.33 & V s. & 100 & 9.57 & 9.57 & S. \\
\hline 102 & 5.60 & 5.61 & $\mathrm{~s}$. & 002 & 7.78 & 7.78 & vs. \\
\hline 002 & 5.46 & 5.44 & s. & 111 & 6.16 & 6.16 & vw. \\
\hline 011. & 4.95 & 4.98 & s. & 003 & 5.19 & 5.20 & $\mathrm{~m}$ \\
\hline 202 & 4.79 & 4.79 & $\mathrm{~m}$. & 200 & 4.57 & 4.56 & w. \\
\hline 300 & 4.15 & 4.12 & $\mathrm{~m}$. & 103 & 4.56 & 4.55 & w. \\
\hline 302 & 3.89 & 3.87 & m. & 211 & 4.27 & 4.24 & w. \\
\hline 211 & 3.66 & 3.63 & s. & 202 & 4.07 & 4.07 & vw. \\
\hline 202 & 3.64 & 3.62 & m. & 212 & 3.74 & 3.72 & w. \\
\hline 313 & 3.33 & 3.32 & w. & 203 & 3.52 & 3.54 & s. \\
\hline 400 & 3.12 & 3.12 & s. & 220 & 3.38 & 3.38 & m. \\
\hline 013 & 3.05 & 3.05 & m. & 222 & 3.10 & 3.14 & w. \\
\hline 311 & 3.04 & 3.04 & w. & 005 & 3.09 & 3.09 & $\mathrm{~m}$. \\
\hline 302 & 2.94 & 2.99 & w. & 130 & 3.02 & 3.03 & w. \\
\hline $\begin{array}{l}213 \\
120\end{array}$ & 2.52 & 2.52 & $\mathrm{~m}$. & & & & \\
\hline 500 & 2.50 & 2.50 & w. & & & & \\
\hline 600 & 2.08 & 2.08 & $\mathrm{w}$. & & & & \\
\hline
\end{tabular}

The precipitates of $\beta$-form copper-oxinate 2 hydrate are hexagonal, rhombohedral, or tetragonal form, and the hexagonal form has constant plane angles at $116^{\circ}$ and $128^{\circ}$ (Fig. 1(b)). $\beta$-Form copper- 
oxinate 2 hydrate was occasionally prepared as a large glittering thin crystal. This sample, without being pulverized by a mortar, is packed to a flat plate, so that the crystals are arranged so as to take a fibrous structure as a whole and each flat habit surface comes in parallel to the plane of the plate-like specimen for X-ray diffractometer analysis. Its $\mathrm{X}$-ray diffraction pattern gives only such peaks as correspond to the $(h 00)$ reflection $(h=2 n+1)$ as shown with the dotted line in Fig. 2(a).

A diffraction can be described only for the equator reflections attributable to net planes, which are in parallel to the flat plane of the specimen. In this case, the $(h 00)$ plane of each crystal comes in parallel to the flat plane of this specimen. This suggests that the flat habit surface of hexagonal plate is (100). By geometrically calculating the various plane angles using the cell constants, the plane angles of $116^{\circ}$ and $128^{\circ}$ consist of those planes as shown in Fig. 3(a), and the crystal habit of $\beta$-form copper-oxinate 2 hydrate is shown in Fig. 3(b). The variety in form of precipitates, namely hexagonal, rhombohedral, or tetragonal, depends on the conditions of precipitation, which causes the growth of different planes, but the X-ray diffraction patterns of these precipitates are the same.

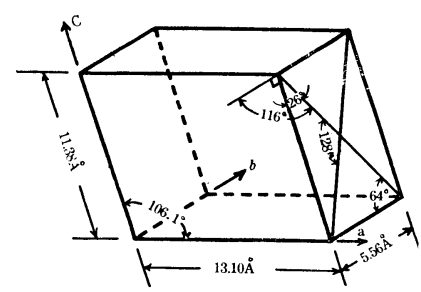

(a)

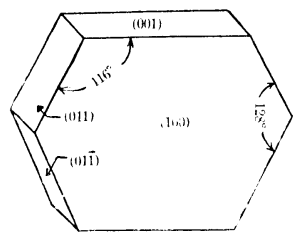

(b)

Fig. 3. (a): Schematic unit cell of $\beta$-form copper-oxinate 2 hydrate (b): Crystal habit of $\beta$-form copper-oxinate 2 hydrate

(b) $\alpha$-Form copper-oxinate 2 hydrate. The X-ray pattern of $\alpha$ form copper-oxinate 2 hydrate is different from that of $\beta$-form and the number of diffraction peaks is smaller than that of $\beta$-form. This fact suggests that the crystal system of unstable $\alpha$-form copper-oxinate 2 hydrate belongs to the higher symmetric system such as orthorhombic, tetragonal, etc.

Now, copper-phthalocyanine precipitate has also dimorphism. The space group of stable $\beta$-form copper-phthalocyanine,,$^{5)}$ in which planar phthalocyanine molecule coordinates around the copper atom as in the case of copper-oxinate, is $P 2_{1} / \alpha$ as in $\beta$-form copper-oxinate 2 hydrate. It has been assumed the $\alpha$-form copper-phthalocyanine belongs to tetragonal system with powder X-ray diffraction by Robinson et al., ${ }^{6)}$ 
and with electron microdiffraction by Suito and Uyeda."

Then, the interplanar spacings of $\alpha$-form copper-oxinate 2 hydrate calculated from X-ray diffraction pattern accord with the Hull and Davey's diagram for tetragonal system at the position of about 1.6 for $c / a$ value. By assuming the following cell constant, $a=b=9.57, c=$ 15.56 $\AA$, $\alpha=\beta=\gamma=90^{\circ}$, and using these constants, interplanar spacings were calculated, which agreed in a $1 \%$ accuracy as shown in Table II.

It has been reported that unstable $\alpha$-form copper-phthalocyanine transforms into $\beta$-form in some organic solvents. ${ }^{78)}$ The crystal structure of copper-oxinate 2 hydrate resembles to that of copperphthalocyanine. It is assumed that unstable $\alpha$-form copper-oxinate 2 hydrate belonging to tetragonal system forms at the outset of formation of precipitate and then transforms into stable $\beta$-form belonging to monoclinic system.

The authors wish to express their hearty thanks to Prof. M. Ishibashi, Faculty of Science, Kyoto University, for his kind advice and also to Mr. N. Uyeda for his useful criticism.

\section{References}

1) M. Ishibashi, E. Suito, and E. Sekido: J. Chem. Soc. Japan, 77, 1836 (1956).

2) E. B. Sandell: Colorimetric Determination of Trace of Metals, 116 (1950).

3) L. L. Beritt, R. T. Cady, Jr., and B. W. Mundy: Acta Cryst., 7, 473 (1954).

4) R. Kruh and C. W. Dwiggins: J. Am. Chem. Soc., 77, 806 (1955).

5) J. M. Robertson: J. Chem. Soc., 1736 (1936); 615 (1935).

6) M. T. Robinson and G. E. Klein: J. Am. Chem. Soc., 74, 6294 (1952).

7) E. Suito and N. Uyeda: Proc. Japan Acad., 32, 182 (1956).

8) N. Uyeda and E. Suito: J. Electromicroscopy, 4, 36 (1956). 